\title{
THE LEVELS OF ${ }^{103}$ Rh POPULATED IN THE DECAY OF ${ }^{103}$ Ru
}

\author{
D. E. RAESIDE, J. J. REIDY and M. L. WIEDENBECK \\ Physics Department, The University of Michigan, Ann Arbor, Michigan $48104{ }^{\dagger}$
}

Received 5 May 1969

Abstract: The gamma-ray spectrum associated with the beta decay of ${ }^{103} \mathrm{Ru}$ has been studied with curved-crystal and $\mathrm{Ge}(\mathrm{L} i)$ spectrometers. The energy measurements obtained from the curvedcrystal spectrometer data are of greater precision than previously reported measurements. Two gamma rays known to belong to the decay of ${ }^{103} \mathrm{Ru}$ but not seen directly by previous investigators in gamma-ray singles spectra were observed, and intensities are given. Coincidence spectra were taken using a $\mathrm{Ge}(\mathrm{Li})-\mathrm{Ge}(\mathrm{Li})$ combination. The data confirm the essential features of previously proposed ${ }^{103} \mathrm{Rh}$ level schemes.

\section{Introduction}

Although the beta decay of ${ }^{103} \mathrm{Ru}$ has been frequently investigated ${ }^{1-8}$ ), there has yet to be a study of the gamma-ray spectrum utilizing spectrometers capable of fully resolving all gamma rays known to belong to this decay. The present investigation was undertaken to fill this gap and to provide precision energy measurements and improved intensity measurements of the gamma rays associated with the decay of ${ }^{103} \mathrm{Ru}$.

\section{Experimental arrangements}

The curved-crystal spectrometer energy measurements reported in this paper were taken on the University of Michigan $2 \mathrm{~m}$ curved-crystal spectrometers. One spectrometer utilized the $\mathrm{Ge}(0 \overline{2} 2)$ planes as diffraction planes, while the other utilized the $Q(310)$ planes. These spectrometers and the experimental techniques associated with their use have been described previously ${ }^{9-11}$ ). Both crystals were calibrated using the $411.795 \pm 0.007 \mathrm{keV}$ gamma ray ${ }^{12}$ ) occurring in the decay of ${ }^{198} \mathrm{Au}$. We have adopted a smaller uncertainty for this gamma-ray energy than the one given in ref. ${ }^{12}$ ). This reflects the smaller uncertainty assigned to the value for the rest mass energy of the electron in the latest least-squares adjustment of the fundamental constants ${ }^{15}$ ). The source used with the curved-crystal spectrometers was a mixture of Ru metal (obtained from Oak Ridge National Laboratory, enriched to $99.53 \%$ in ${ }^{102} \mathrm{Ru}$ ) and

t This work was supported in part by the US Atomic Energy Commission. 
epoxy resin. This source was irradiated in The University of Michigan Ford Nuclear Reactor (thermal neutron flux $\approx 3 \times 10^{13}$ neutrons $/ \mathrm{sec} \cdot \mathrm{cm}^{2}$ ) for a period of approximately 12 weeks; its activity at the end of this period was about $0.6 \mathrm{Ci}$. Energy resolutions of the two spectrometers were about equal: $\Delta E(\mathrm{FWHM})=\left(2.5 \times 10^{-5}\right)$ $\left(E^{2} / n\right) \mathrm{keV}$, where $n$ is the order of reflection and $E$ the gamma-ray energy in keV.

The detector used for energy and intensity measurements was an Ortec $32 \mathrm{~cm}^{3}$ coaxial $\mathrm{Ge}(\mathrm{Li})$ detector coupled to a Canberra $1408 \mathrm{C}$ preamplifier and a Tennelec TC200 amplifier. Data were accumulated in a Scipp 1600-channel analyser. The nonlinearities in the system were accounted for using the method of Donnelly et al. ${ }^{14}$ ). A relative efficiency calibration ${ }^{14}$ ) for the system was obtained for the range 90-1400 $\mathrm{keV}$ by utilizing sources for which the relative emission of pairs of gamma rays are precisely known; namely ${ }^{180 \mathrm{~m}} \mathrm{Hf},{ }^{108 \mathrm{~m}} \mathrm{Ag},{ }^{22} \mathrm{Na},{ }^{60} \mathrm{Co},{ }^{160} \mathrm{~Tb}$ and ${ }^{46} \mathrm{Sc}$. For gammaray intensities in the energy range below $90 \mathrm{keV}$ a modified pair-point method ${ }^{15}$ ) was used. In this method, a comparison is made of the relative peak areas of the gamma rays de-exciting a single level and the $\mathrm{X}$-rays which follow internal conversion. By using three sources $\left({ }^{137} \mathrm{Cs},{ }^{139} \mathrm{Ce}\right.$ and $\left.{ }^{160} \mathrm{~Tb}\right)$, the relative efficiency calibration was extended to $30 \mathrm{keV}$. The source material used for the $\mathrm{Ge}(\mathrm{Li})$ spectrometer was taken from the same enriched sample used in the fabrication of the curved-crystal spectrometer source. A few grains of this finely powdered ruthenium metal were encapsulated in a quartz vial and irradiated in The University of Michigan Ford Nuclear Reactor for about 12 weeks. The source was allowed to cool for about 16 weeks before spectra were taken. $\mathrm{A} \mathrm{Ge}(\mathrm{Li})$ spectrometer source was prepared by placing some of the radioactive material into a cavity in a lucite disk. The activity of this source was about $3 \mu \mathrm{Ci}$.

The source used for the measurement of gamma-ray energies and intensities on the $\mathrm{Ge}(\mathrm{Li})$ spectrometer was also used for the gamma-gamma coincidence study. The total spectrum channel of the coincidence system consisted of the coaxial $\mathrm{Ge}(\mathrm{Li})$ detector, preamplifier and amplifier described above; the gate channel consisted of a $40 \mathrm{~cm}^{3}$ trapezoidal $\mathrm{Ge}(\mathrm{Li})$ detector coupled to a Nuclear Diodes 101 preamplifier, a Tennelec TC200 amplifier and TC250 biased amplifier and stretcher. The resolution of both detectors was about $2.5 \mathrm{keV}$ at $662 \mathrm{keV}$. The coincidence electronics was of the fast-slow type. Leading-edge time was used for both channels. A resolving time $2 \tau$ of about $110 \mathrm{~ns}$ was used for all runs. Two coincidence spectra were taken for each run, one with the gate set on the photopeak of interest and the other with the gate set on the energy region immediately adjacent to it. The two spectra were routed to 800channel subgroups of the Scipp 1600-channel analyser.

\section{Results}

The results of the energy and intensity measurements are presented in table 1 . The values of $E_{\gamma}(40 \mathrm{keV}), E_{\gamma}(53 \mathrm{keV}), E_{\gamma}(295 \mathrm{keV}), E_{\gamma}(497 \mathrm{keV})$ and $E_{\gamma}(610 \mathrm{keV})$ are curved-crystal spectrometer measurements; the uncertainties shown are a combina- 
tion of the uncertainties associated with the data and the uncertainties associated with the curved-crystal spectrometer calibration. The values of $E_{\gamma}(444 \mathrm{keV})$ and $E_{\gamma}(557$ $\mathrm{keV}$ ) are the weighted averages of values obtained from the curved-crystal spectrometer and the $\mathrm{Ge}(\mathrm{Li})$ spectrometer (calibrations of the $\mathrm{Ge}(\mathrm{Li})$ spectra were performed

TABLE 1

Energies and intensities of gamma-ray transitions occurring in the decay ${ }^{103} \mathrm{Ru} \stackrel{\beta}{\rightarrow}{ }^{103} \mathrm{Rh}$

\begin{tabular}{cc}
\hline Energy $(\mathrm{keV})$ & Relative gamma-ray intensity \\
\hline $39.755 \pm 0.012$ & $0.037 \pm 0.009$ \\
$53.275 \pm 0.010$ & $0.34 \pm 0.06$ \\
$294.72 \pm \mathbf{0 . 1 2}$ & $0.30 \pm 0.03$ \\
$443.77 \pm 0.12$ & $0.34 \pm 0.03$ \\
$497.080 \pm 0.013$ & $100 \quad 0.88 \pm 0.07$ \\
$557.11 \pm 0.13$ & $6.24 \pm 0.31$ \\
\hline
\end{tabular}

using our curved-crystal spectrometer measurements of the 497 and $610 \mathrm{keV}$ gamma rays); the uncertainties are a combination of the uncertainties associated with the data and the uncertainties associated with calibration. Typical Ge(Li) spectra are shown in figs. 1 and 2. These spectra clearly show the peaks due to the $40 \mathrm{keV}$ and $444 \mathrm{keV}$ gamma rays which have been known ${ }^{1-3}$ ) to occur in the decay of ${ }^{103} \mathrm{Ru}$. This is the first report of their observation as separate peaks in gamma-ray singles spectra.

Several gamma rays not observed in the present work have been reported by previous investigators as belonging to the decay of ${ }^{103} \mathrm{Ru}$. We estimate the following upper limits for some of them (relative to the intensity of the $497 \mathrm{keV}$ gamma ray, $\left.I_{\gamma}(497 \mathrm{keV}) \equiv 100\right): I_{\gamma}(65 \mathrm{keV})<0.01, I_{\gamma}(323 \mathrm{keV})<0.08$ and $I_{\gamma}(362 \mathrm{keV})<0.05$

A partial summary of the results of the gamma-gamma coincidence study is shown in table 2. Allowance has been made for contributions due to chances and Compton scattered photons in the gated region.

A level scheme which is consistent with the result of the present investigation is shown in fig. 3. This level scheme is in essential agreement with the work of previous investigators ${ }^{1-8}$ ). The ground state spin and parity as well as the spins and parities of the $40 \mathrm{keV}$ and $295 \mathrm{keV}$ levels appear to be fairly well established. The available data regarding the spin and parity of the $537 \mathrm{keV}$ level are not at all conclusive. Manthuruthil et al. ${ }^{1}$ ) have recently suggested $\frac{7}{2}^{+}$and $\frac{7}{2}^{-}$as possible spin and parity assignments for the 93 and $650 \mathrm{keV}$ levels, respectively.

Several possible interlevel transitions can be placed in the level scheme of fig. 3. Curved-crystal spectrometer searches were made for two of these possible transitions (113 and $202 \mathrm{keV}$ ), but neither of them was observed. We estimate an intensity upper limit for each of these possible gamma rays of 0.03 (relative to the intensity of the $497 \mathrm{keV}$ gamma ray, $\left.I_{\gamma}(497 \mathrm{keV}) \equiv 100\right)$. 


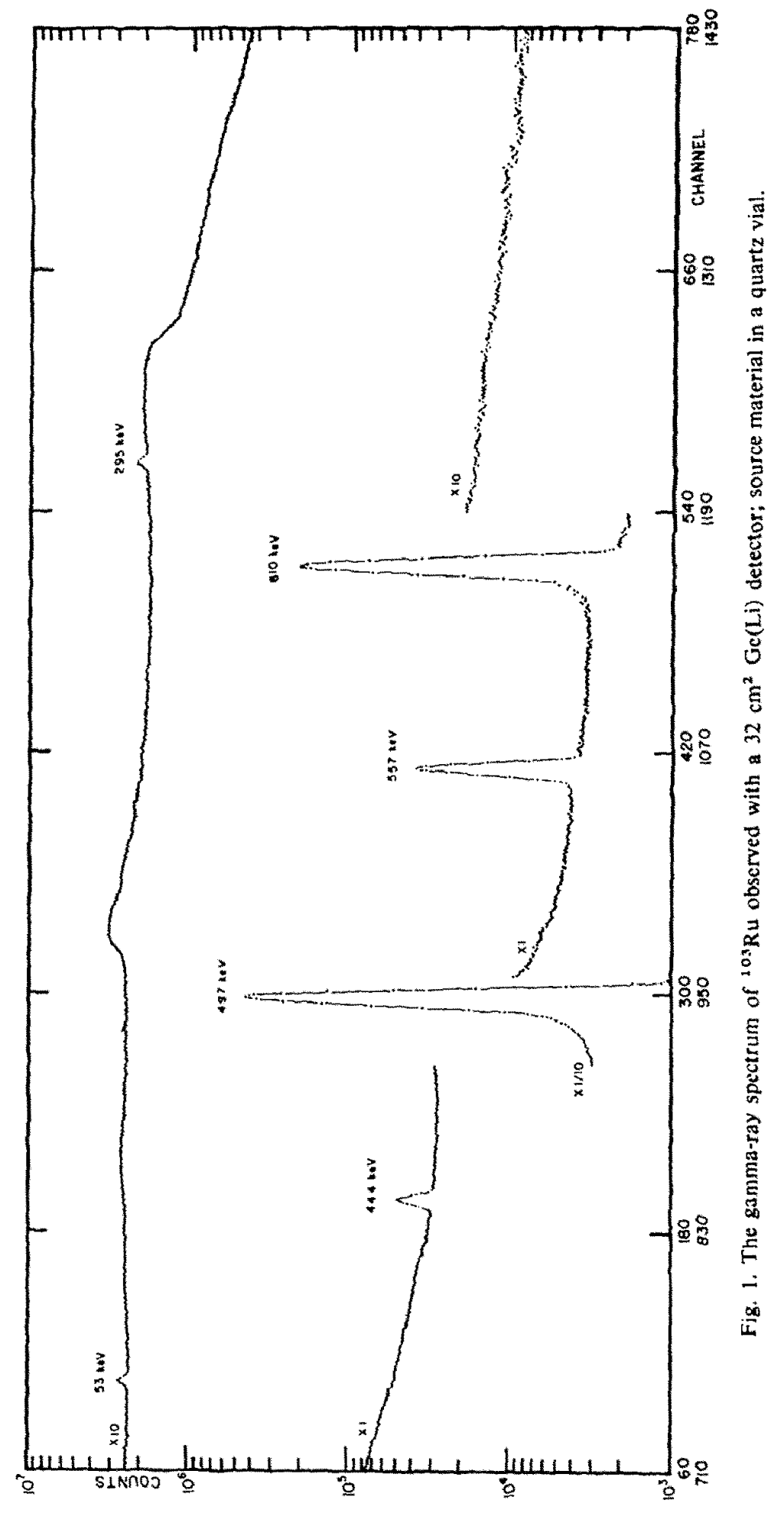




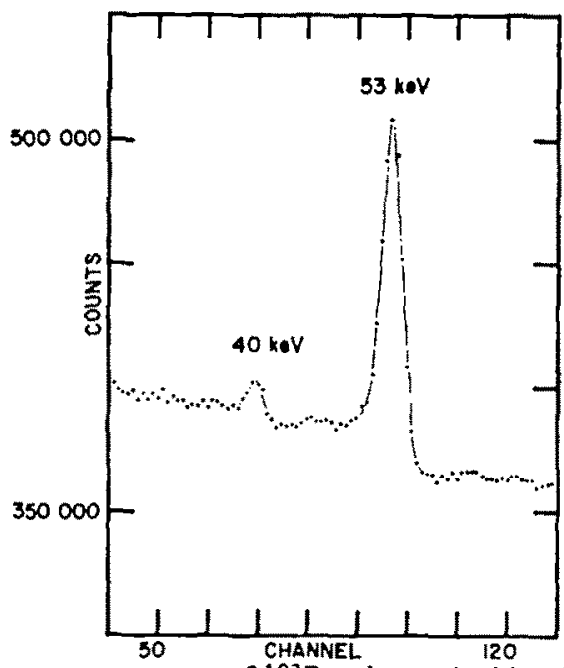

Fig. 2. The two low-energy gamma rays of ${ }^{103} \mathrm{Ru}$ observed with a $32 \mathrm{~cm}^{3} \mathrm{Ge}(\mathrm{Li})$ detector; source material on a lucite disk. Note that the vertical scale is linear.

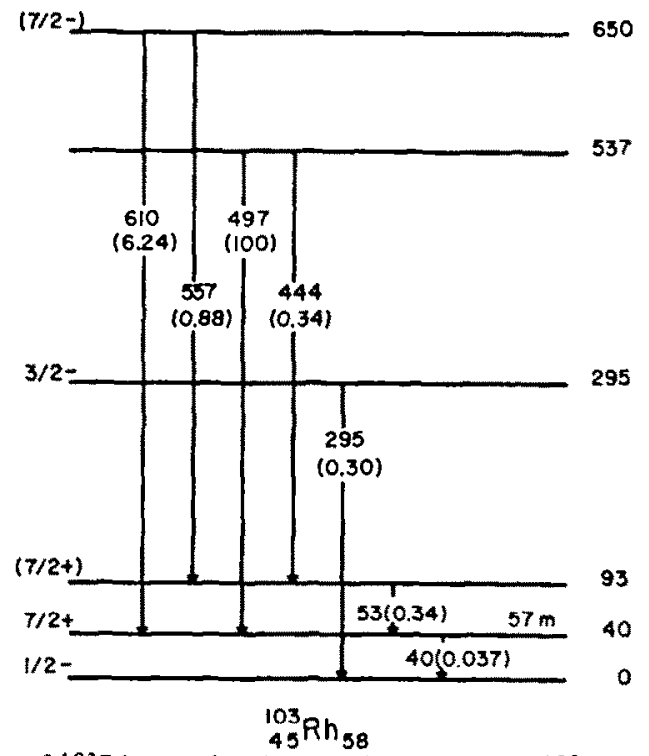

Fig. 3. The level scheme of ${ }^{103} \mathrm{Rh}$ populated in the beta decay of ${ }^{103} \mathrm{Ru}$. Gamma-ray energies are given in keV; relative gamma-ray intensities are enclosed by parentheses.

TAble 2

Gamma-gamma prompt coincidence results

\begin{tabular}{cl}
$\begin{array}{c}\text { Gate } \\
(\mathrm{keV})\end{array}$ & \multicolumn{1}{c}{$\begin{array}{c}\text { Prompt coincident gamma rays } \\
(\mathrm{keV})\end{array}$} \\
\hline 295 & none \\
444 & 53 \\
497 & none \\
557 & 53 \\
610 & none
\end{tabular}




\section{Conversion coefficients}

It has been pointed out ${ }^{1}$ ) that the $53 \mathrm{keV}$ transition occurring in the decay of ${ }^{103} \mathrm{Ru}$ is probably pure M1. If we assume that this is the case, then we can compute $\mathrm{K}$-shell internal conversion coefficients by utilizing the gamma-ray intensities of the present work, the electron intensities of ref. ${ }^{1}$ ) and the theoretical value of the $\mathrm{K}$-shell internal conversion coefficient for the $53 \mathrm{keV}$ transition. We find by interpolation of the values given by Hager and Seltzer $\left.{ }^{16}\right)$ that $\alpha_{K}(53.275 \mathrm{keV}, Z=45 ; \mathrm{M} 1)=1.82$. With this value as a normalization constant, we calculate the following conversion coefficients:

$$
\begin{aligned}
& \alpha_{\mathbf{K}}(295 \mathrm{keV})=0.016 \pm 0.005 \\
& \alpha_{\mathbf{K}}(444 \mathrm{keV})=0.012 \pm 0.003 \\
& \alpha_{\mathbf{K}}(497 \mathrm{keV})=0.0058 \pm 0.0015 \\
& \alpha_{\mathbf{K}}(557 \mathrm{keV})=0.0068 \pm 0.0018 \\
& \alpha_{\mathbf{K}}(610 \mathrm{keV})=0.0037 \pm 0.0009
\end{aligned}
$$

These values are in good agreement with those deduced by Manthuruthil et al. ${ }^{1}$ ).

We wish to thank M. A. Ludington for providing the relative efficiency calibration of the $\mathrm{Ge}(\mathrm{Li})$ detector and S. R. Weiner and D. H. Dye for help in data acquisition and reduction.

\section{References}

1) J. C. Manthuruthil, H. J. Hennecke and C. R. Cothern, Phys. Rev. 165 (1968) 1363

2) V. R. Potnis, E. B. Nieschmidt, C. E. Mandeville, L. D. Ellsworth and G. P. Agin, Phys. Rev. $146(1966) 883$

3) A. Mukerji, D. N. McNelis and J. W. Kane, Nucl. Phys. 67 (1965) 466

4) S.-E. Karlsson, O. Bergman and W. Scheuer, Ark. Fys. 27 (1964) 61

5) S. I. H. Naqvi and B. G. Hogg, Phys. Rev. 128 (1962) 357

6) H. H. Forster and A. Rosen, Nuovo Cim. 1 (1955) 972

7) B. de Raad, W. C. Middelkoop, B. van Nooijen and P. M. Endt, Physica 20 (1954) 1278

8) B. Saraf, Phys. Rev. 97 (1955) 715

9) J. J. Reidy and M. L. Wiedenbeck, Nucl. Instr. 33 (1965) 213

10) J. J. Reidy and M. L. Wiedenbeck, Nucl. Phys. 70 (1965) 518

11) J. J. Reidy and M. L. Wiedenbeck, Nucl. Phys. 79 (1966) 193

12) G. Murray, R. L. Graham and J. S. Geiger, Nucl. Phys. 63 (1965) 353

13) E. R. Cohen and J. W. M. DuMond, Revs. Mod. Phys. 37 (1965) 537

14) D. P. Donnelly, H. W. Baer, J. J. Reidy and M. L. Wiedenbeck, Nucl. Instr. 57 (1967) 219

15) D. P. Donnelly and M. L. Wiedenbeck, Nucl. Instr. 64 (1968) 26

16) R. S. Hager and E. C. Seltzer, Nucl. Data 4A (1968) 59 